\title{
APPLICATION OF POLYNOMIAL CHAOS EXPANSION TO RELIABILITY ANALYSIS OF PRESTRESSED CONCRETE ROOF GIRDERS
}

\author{
L. Novák ${ }^{*}$, D. Novák ${ }^{* *}$, O. Slowik ${ }^{* * *}$
}

\begin{abstract}
The paper is focused on the application of the surrogate model to reliability analysis of real structure. First, the basic theory of polynomial chaos expansion (PCe) is described. Usage of the PCe for moment and sensitivity analysis is then presented, which can be done efficiently by post-processing of explicit function without any additional computational demands. Implementation of theory into new software tool and its application on prestressed concrete roof girder failing in shear is presented in the last part of the paper. As can be seen in the example, PCe for reliability analysis represents very strong and efficient tool, especially for variance based sensitivity analysis and estimation of statistical moments.
\end{abstract}

Keywords: structural reliability, Polynomial chaos expansion, surrogate model, sensitivity analysis

\section{Introduction}

The mathematical model of physical problem $\mathcal{M}(\mathbf{X})$ is represented by a function of set of input variables. In practical applications, this function is often in implicit form solved by non-linear finite element method (NLFEM). NLFEM is very accurate method to solve mathematical models but to obtain realistic results it is necessary to use random input variables described by probability distribution. The combination of structural reliability methods and NLFEM is computationally time consuming, especially in case of huge mathematical model with many random input variables. One of the methods to reduce the computational requirements is the approximation of NLFEM by explicit function - surrogate model. There are several types of surrogate models described in scientific papers: Polynomial chaos expansion, krigging, support vector machine or artificial neural networks. The main steps are common for all the methods. First, several calculations of the original mathematical model is performed with different realizations of input random vector generated by Monte Carlo type simulation techniques. The results of simulations are used as "training" set for the creation of approximation function. Last and the most important step is the estimation of error of the approximation, this can be done by various methods. The paper describes the surrogate model via polynomial chaos expansion (PCe) as described by Sudret et al. (2012).

\section{Polynomial chaos expansion}

Let's assume that random model response $Y=\mathcal{M}(\mathbf{X})$ has a finite variance, thus chaos representation is according to Soize and Ghanem (2004) following:

$$
Y=\mathcal{M}(\mathbf{X})=\sum_{\alpha \in \mathbb{N}^{M}} \beta_{\alpha} \Psi_{\alpha}(\mathbf{X})
$$

where $M$ is the number of input random variables, $\beta_{\alpha}$ are unknown deterministic coefficients and $\Psi_{\alpha}$ are multivariate basis functions orthonormal with respect to the joint probability density function of $\mathbf{X}$. In this

\footnotetext{
Lukáš Novák: Institute of structural mechanics, Brno University of Technology, Veveří 331/95; 602 00, Brno; CZ, Lukas.Novak4@vut.cz

** Prof. Ing. Drahomír Novák, DrSc.: Institute of structural mechanics, Brno University of Technology, Veveř́ 331/95; 602 00, Brno; CZ, novak.d@fce.vutbr.cz

**** Ing. Ondřej Slowik.: Institute of structural mechanics, Brno University of Technology, Veveří 331/95; 602 00, Brno; CZ
} 
paper the standard normal input variables $\boldsymbol{\xi}$ are assumed, thus normalized Hermite polynomials are used as basis functions. In case of non-Gaussian or correlated input variables, the Nataf transformation must be performed. More informations can be found in (Nataf, 1962).

\subsection{Estimation of PCe coefficients}

For practical implementation of the PCe into the software tool, the expansion must be truncated to get only finite number of terms. Commonly used truncated set of basis functions $\mathcal{A}^{M, p}$ is dependent on maximal order of used polynomials $p \leq|\alpha|=\sum_{i=1}^{M} \alpha_{i}$ and $M$ :

$$
\mathcal{A}^{M, p}=\left\{\alpha \in \mathbb{N}^{M}:|\alpha| \leq p\right\}
$$

In this paper, the least-square regression method is used to compute the coefficients as described in (Berveiller et al., 2004). First the set of $n$ realizations of random input vector $\mathcal{X}$ called experimental design (ED) is created by Monte Carlo techniques and the vector of corresponding results $\mathcal{Y}$ obtained by NLFEM. The estimates of coefficients are thus given by following equation where $\boldsymbol{\Psi}$ is data matrix calculated from the evaluation of the basis polynomials onto each point in the ED:

$$
\boldsymbol{\beta}^{L . S}=\left(\boldsymbol{\Psi}^{T} \boldsymbol{\Psi}\right)^{-1} \boldsymbol{\Psi}^{T} \mathcal{Y}
$$

\subsection{Validation of PCe}

It is common to compute determination coefficient when using surrogate model. However, this approach may lead to overfitting, especially when number of points in ED is low. Improvement of the standard method is called leave-one-out (LOO) cross validation. The idea is to use different sets of points to build a PCe and to compute the error. LOO sets one point apart from the full ED and builds a PCe from remaining points. This process is repeated for every point of ED. But in case of using PCe as surrogate model, it is possible to build just one PCe with full original ED and estimate LOO error analytically as follows:

$$
Q^{2}=1-\frac{\frac{1}{n} \sum\left(\frac{\mathcal{M}\left(x^{(i)}\right)-\left(\mathcal{M}^{P C e}\left(x^{(i)}\right)\right.}{1-h_{i}}\right)^{2}}{\operatorname{Var}[\mathcal{Y}]}
$$

where $h_{i}$ represents $i$-th diagonal term of matrix $\boldsymbol{\Psi}\left(\boldsymbol{\Psi}^{T} \boldsymbol{\Psi}\right)^{-1} \boldsymbol{\Psi}^{T}$. As a conclusion, the $Q^{2}$ can be obtained posteriori without any additional computational demand.

\subsection{Post-processing of coefficients}

Due to the orthonormality of the PCe basis some informations about mathematical model can be computed just by post-processing of estimated coefficients, specifically statistical moments and sensitivity analysis. The mean $\mu_{\tilde{Y}}$ and variance $\sigma_{\tilde{Y}}^{2}$ of the surrogate model can be easily computed:

$$
\begin{gathered}
\mu_{\tilde{Y}}=\mathbb{E}[\tilde{Y}]=\mathbb{E}\left[\sum_{\alpha \in \mathcal{A}} \beta_{\alpha} \Psi_{\alpha}(\boldsymbol{\xi})\right]=\beta_{0} \\
\sigma_{\tilde{Y}}^{2}=\operatorname{Var}[\tilde{Y}]=\mathbb{E}\left[\left(\tilde{Y}-\beta_{0}\right)^{2}\right]=\sum_{\substack{\alpha \in \mathcal{A} \\
\alpha \neq 0}} \beta_{\alpha}^{2}
\end{gathered}
$$

Variance-based sensitivity analysis can be computed similarly, more informations can be found in (Sudret, 2008). Total Sobol' indices $S_{i}^{T}$ can be estimated by following equation:

$$
S_{i}^{T}=\sum_{\alpha \in \mathcal{A}_{i}^{T}} \frac{\beta_{\alpha}^{2}}{\operatorname{Var}[\tilde{Y}]} \quad \mathcal{A}_{i}^{T}=\left\{\alpha \in \mathbb{N}^{M}: \alpha_{i}>0\right\}
$$




\section{PCe software tool}

Presented theory of PCe was implemented into SW tool cooperating with SW FReET (Novák et al., 2014). Main purpose of SW tool is the creation of PCe surrogate model as efficient as possible and easily applicable for any problem. Thus algorithm least angle regression (LAR) described by Efron et al. (2004) was implemented for reduction of number of basis functions. SW tool is able to automatically build PCe for target accuracy given by $Q^{2}$ or the best possible variant due to initial ED. Surrogate model is used for statistical moment and sensitivity analysis and can be exported to SW FReET for advanced reliability analysis.

\section{Practical example}

PCe software tool was used for moment and sensitivity analysis of ultimate shear capacity of prestressed reinforced concrete roof girder described by Slowik et al. (2015). Material characteristics of concrete were obtained by laboratory experiments and are presented in Tab.1, where $f_{c}$ is compressive strength of concrete, $f_{t}$ is tensile strength, $G_{f}$ represents fracture energy and $E$ is Young's modulus. Statistical parameters of tendons and steel were taken from (Probabilistic model code, 2001). Full stochastic model contains 14 correlated random variables, more details can be found in (Slowik et al., 2017).

Tab. 1: Stochastic model of concrete girder

\begin{tabular}{ccccc}
\hline Prameter & Mean & CoV $[\%]$ & PDF & Unit \\
\hline$f_{c}$ & 77 & 16.4 & Lognormal & {$[M P a]$} \\
$f_{t}$ & 3.9 & 20.6 & Lognormal & {$[M P a]$} \\
$E$ & 34.8 & 20.6 & Lognormal & {$[G P a]$} \\
$G_{f}$ & 219.8 & 32.8 & Lognormal & {$\left[\mathrm{J} \cdot \mathrm{m}^{-2}\right]$} \\
Density & 0.0023 & 4 & Normal & {$\left[\mathrm{kton} / \mathrm{m}^{3}\right]$} \\
\hline
\end{tabular}

Surrogate model was accomplished with maximal order of polynomial $p=3$ and ED contains 31 points generated by Latin Hypercube Sampling. Least angle regression algorithm was used to reduce the size of truncated set of basis functions from 455 terms to 9 basis functions. Basic informations about surrogate model are presented in the following lists:

\section{PCe SETTINGS:}

- Maximal order of polynomial: 3

- Target error estimation Leave-one-out: 0,95

- Sparse PCe by LARS: True

- Full size of polynomial chaos basis:455

- Size of experimental design: 31

\section{SURROGATE MODEL:}

- Mean value: $195,8303 k N$

- Variance value: $699,8746 k N^{2}$

- Coefficient of determination $R^{2}: 0,9630$

- Leave-one-out error $Q^{2}$ : 0,9093

- Size of sparse LAR basis: 9

As can be seen, LAR drastically reduced the number of PCe basis functions, thus ED containing only 31 point was sufficient to obtain $91 \%$ accuracy of approximation. Statistical parameters are in compliance with mean and variance of $\mathrm{ED}-$ mean $=198.5$ and variance $=683.5$.

Sensitivity analysis in terms of Sobol' indices can be seen in the Fig.1. Important variables are concrete material characteristics and uncertainty of calculation of losses in prestressing. Note that, for computation of Sobol' indices were only 31 points in ED needed, which is very low number in comparison with Monte Carlo techniques. 


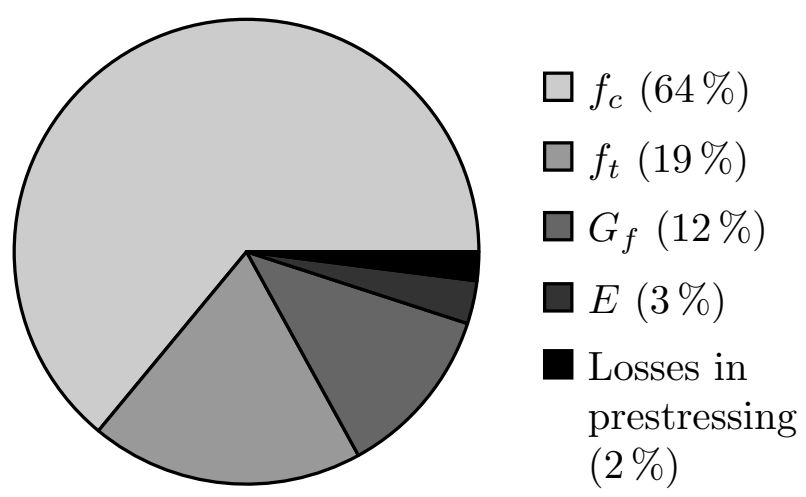

Fig. 1: Sobol' indices

\section{Conclusions}

In the paper, implementation of PCe into the software tool and its application were presented. As can be seen in practical example, LAR algorithm in combination with PCe is a strong tool for reliability analysis of computational models solved by NLFEM. Surrogate model was used for moment and sensitivity analysis, which can be obtained by post-processing of PCe without any additional computational demand. Thus PCe is very efficient way to obtain Sobol' indices and statistical moments of function, which can be used for reliability-based design of structures.

\section{Acknowledgments}

The authors thanks for support from Czech Science Agency projects: PCe theory has been worked out under project No. 18-13212S (RESUS) and applied for example developed under research in project No. 17$02862 \mathrm{~S}$.

\section{References}

Berveiller, M., Sudret, B. and Lemaire, M. (2004) Presentation of two methods for computing the response coefficients in stochastic finite element analysis. Proc. 9th ASCE Specialty Conference on Probabilistic Mechanics and Structural Reliability, Albuquerque, USA.

Efron, B., Hastie, T. and Johnstone, I. (2004) Least angle regression. The Annals of Statistics, 32: 407-499.

JCSS (2001) JCSS Probabilistic Model Code. Joint Committee on Structural Safety, iSBN 978-3-909386-79-6.

Nataf, A. (1962) Détermination des distributions de probabilité dont les marges sont données. Comptes Rendus de l'Académie des Sciences, 225: 42-43.

Novák, D.; Vořechovský, M. and Teplý, B. (2014) FReET: Software for the statistical and reliability analysis of engineering problems and FReET-D: Degradation module. Advances in Engineering Software, 225: 42-43.

Slowik, O., Novák, D., Krug, B. and Strauss, A. (2015) Shear failure of pre-stressed concrete T-shaped girders: Experiment and nonlinear modeling. In IABSE 2015 conference of International Association for Bridge and Structural Engi-neering, DOI: https://doi.org/10.2749/222137815818358411, Geneva, Switzerland.

Slowik, O.; Novák, D. Novák, L. and Strauss A. (2017) Shear Resistance of Prestressed Girders: Probabilistic Design. In 14th International Probabilistic Workshop, ISBN 978-3-319-47886-9.

Soize, C. and Ghanem, R. (2004) Physical systems with random uncertainties: chaos representations with arbitrary probability measure. J. Sci. Comput, 26(2), 395-410.

Sudret, B. (2008) Global sensitivity analysis using polynomial chaos expansions. Reliab Eng and System Safety, 93: p. $964-979$.

Sudret, B., Berveiller, M. and Lemaire, M. (2012) A stochastic finite element procedure for moment and reliability analysis. Europ journal of Comput mechanics, 15:7-8, 825-866. 